\title{
Metal Foams: Linking Dynamic CT Results to Simulation and Modeling
}

Andreas Grießer ${ }^{1}$, Martina Hümbert ${ }^{2}$, Sebastian Rief $^{2}$, Wesley De Boever ${ }^{3}$ and Luke Hunter ${ }^{4}$

${ }^{1}$ Math2Market GmbH, United States, ${ }^{2}$ Math2Market GmbH, Germany, ${ }^{3}$ Tescan, Belgium, ${ }^{4}$ Tescan, United States

Metal foams are ever-present and used in a wide range of applications, from energy dissipation (safety) to energy saving (lightweighting in automobile industry). The mechanical characteristics of metal foams and their response to external load need to be well understood when they are an integral part of a structure.

For a deeper understanding, we used lab-based dynamic computed tomography (CT) provided by Tescan to visualize uninterrupted compression of aluminum[1] metal foams. These results were coupled to mechanical simulations of the same samples done with the simulation software GeoDict ${ }^{\circledR}$. The dynamic CT reveals the different modes of deformation, like buckling or bending of struts and collapsing of cells. Moreover, the displacement and deformation of pores can be analyzed and quantified once the scan is imported into the simulation software.

Simulating a compression of $35 \%$ is possible thanks to a voxel based FFT solver[2][3] in GeoDict@. By comparing simulation and scan, the simulation can be verified in two ways: first, the load curves show whether the stresses in the foam are similar in simulation and experiment; second, the different deformation modes observed in the simulation can be compared to the deformation modes in the scan, as well as the evolution of pore shapes with increasing compression.

Two samples from the same metal foam are tested, analyzed, and simulated. Data collected from the first sample is used to first analyze and determine material characteristics, which are, in turn, applied as input in a compression simulation of a second sample. The second sample is also physically tested with dynamic CT to provide further verification and improvement of the simulation and modeling results. By comparing the dynamic CT images, a cross-verification of simulation setup and experimental setup is possible, providing further confidence in the results.

In a next step, the verified simulation setup will be used not only on a digital twin obtained by importing a CT scan, but also on a statistical digital twin of a foam which is digitally generated based on the geometrical analysis of the scan. This opens the possibility to create digital metal foam structures with new properties and to predict their deformation. Thus, the GeoDict ${ }^{\circledR}$ software can be used efficiently and cost-effectively in industrial settings to perform digital parameter studies without the need of manufacturing the foam. 

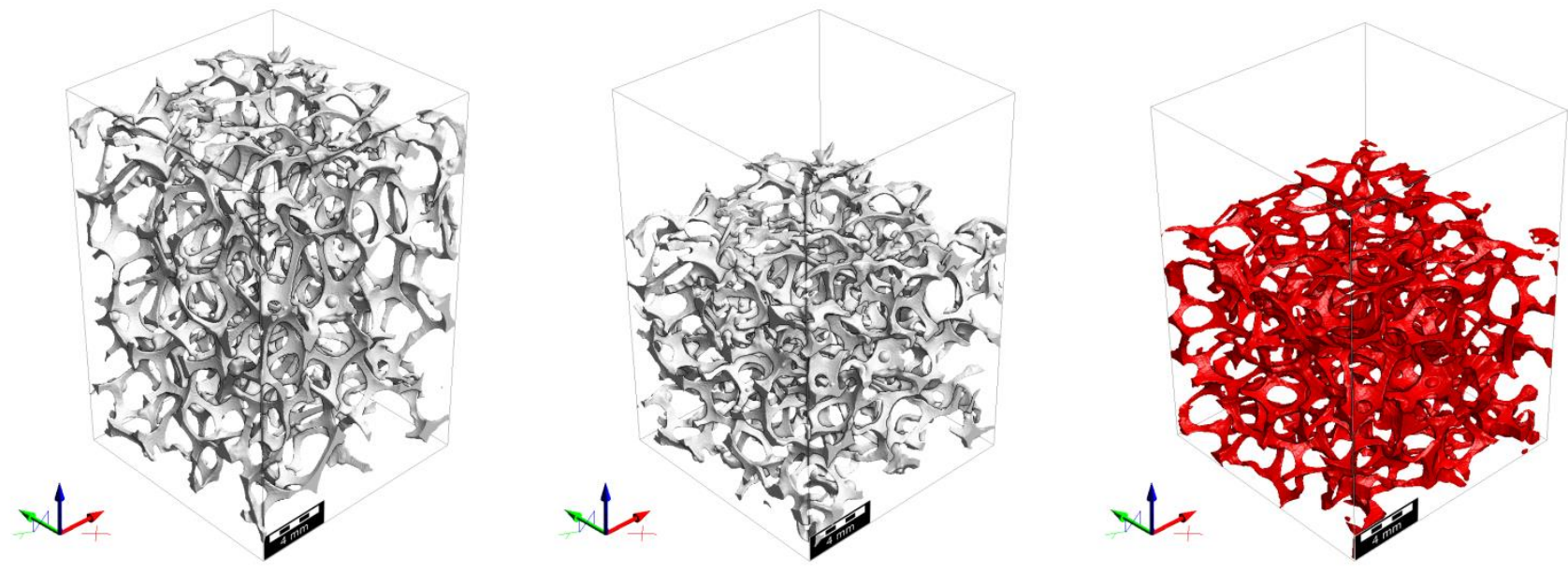

Figure 1. Segmented CT scan before compression, at 35\% compression and simulation result at $35 \%$ compression
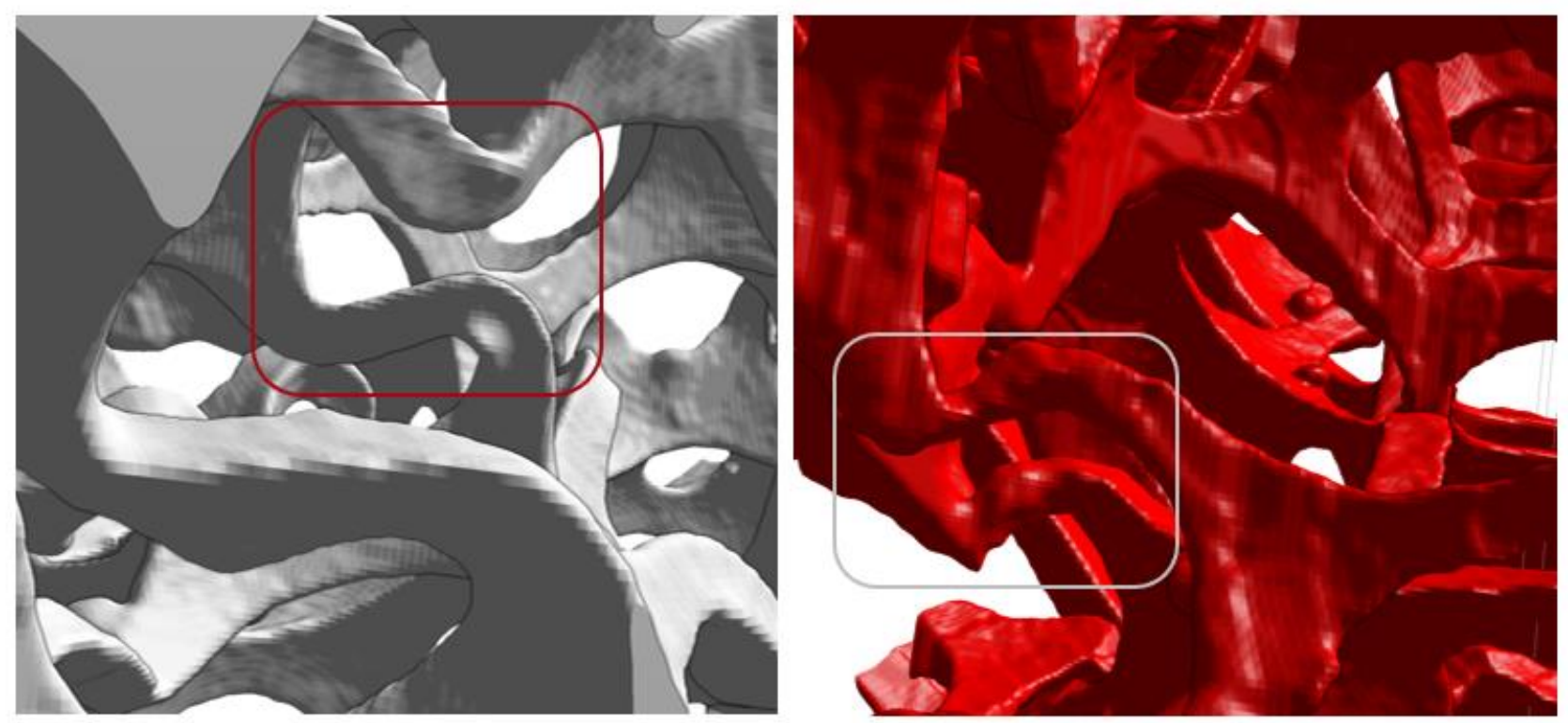

Figure 2. Buckling of struts in experiment and simulation

\section{References}

[1] http://www.steelnumber.com/en/steel_alloy_composition_eu.php?name_id=1014

[2] Use of composite voxels in FFT-based homogenization; Matthias Kabel, Dennis Merkert, Matti Schneider; Computer Methods in Applied Mechanics and Engineering, Volume 294 (2015), pp. 168-188 [3] The composite voxel technique for inelastic problems; M. Kabel, A. Fink, M. Schneider; Computer Methods in Applied Mechanics and Engineering, Volume 322 (2017), pp 396-418 